\title{
A note on entire functions sharing a finite set with their difference operators
}

\author{
Jianming $\mathrm{Qi}^{1,2^{*}}$, Yanfeng Wang ${ }^{3}$ and Yongyi $\mathrm{Gu}^{4}$
}

\section{*Correspondence:} qijianmingsdju@163.com

'Business College, Shanghai Dianji University, Shanghai, P.R. China

${ }^{2}$ Department of Physics and Mathematics, University of Eastern Finland, Joensuu, Finland Full list of author information is available at the end of the article

\begin{abstract}
In this note, we will show that an entire function is equal to its difference operator if it has a growth property and shares a set, where the set consists of two entire functions of smaller orders. This result generalizes a result of Li (Comput. Methods Funct. Theory 12:307-328, 2012 and partially answers Liu's (J. Math. Anal. Appl. 359:384-393, 2009) question.
\end{abstract}

MSC: Primary 30D35; secondary 39A10; 39B32

Keywords: Uniqueness theorems; Entire function; Difference operators; Share set

\section{Introduction and main result}

A set is called a unique range set (URSE) for a certain class of entire functions if each inverse image of the set uniquely determines a function from the given class. Let $S$ be a finite set of some entire functions and $f$ an entire function. Then, a set $E(f, S)$ is defined as

$$
E(f, S)=\{(z, m) \in \mathbb{C} \times Z, f(z)-a(z)=0 \text { with multiplicity } m, a \in S\} .
$$

Assume that $g$ is another entire function. We say that $f$ and $g$ share $S C M$ if $E(f, S)=E(g, S)$. Thus, a set $S$ is called URSE if $E(f, S)=E(g, S)$ where $f$ and $g$ are two entire functions; then $f=g$.

The first example of a URSE was given by Gross and Yang [3], who considered the zero set of equation $z+e^{z}=0$. In view of the fact that this set has infinitely many elements, it is natural to ask whether there exists a finite unique range set or not; the question is proposed by Gross in [4]. In 1995, Yi [5] gave a straightforward answer to Gross' question and found the URSE $w^{n}+a w^{m}+b=0$, where $n>2 m+4$ and $a, b$ satisfy a certain condition. Since then, there have been many efforts to study the problem of constructing unique range sets; see e.g. $[6,7,24]$. There is another study direction on the URSE of entire functions, which is to seek a set $S$ such that if $E(f, S)=E\left(f^{\prime}, S\right)$, then $f=f^{\prime}$ for an entire function $f$. Li and Yang [8] deduced that if $E(f, S)=E\left(f^{\prime}, S\right)$ with $S$ consisting of two distinct constants, then $f$ has specific forms. Later, based on the theory of the normal family, Fang and Zalcman [9] answered the question by proving that there exists a finite set $S$ including three elements such that if $E(f, S)=E\left(f^{\prime}, S\right)$, then $f=f^{\prime}$.

In recent years, the Nevanlinna characteristic of $f(z+\omega)$, the value distribution theory for difference polynomials, the Nevanlinna theory of the difference operator and the difference analogue of the lemma on the logarithmic derivative had been established; see

(c) The Author(s) 2019. This article is distributed under the terms of the Creative Commons Attribution 4.0 International License (http://creativecommons.org/licenses/by/4.0/), which permits unrestricted use, distribution, and reproduction in any medium, provided you give appropriate credit to the original author(s) and the source, provide a link to the Creative Commons license, and indicate if changes were made. 
e.g. $[2,10,11,16-23]$. Due to these theories, there has been recent study of whether the derivative $f^{\prime}$ can be replaced by the difference operator $\Delta_{c} f(z)=f(z+c)-f(z)$ in the above question. In 2009, Liu [2] considered the problem and obtained the result as follows.

Theorem A Suppose that a is a nonzero complex number, and $f$ is a transcendental entire function with finite order. If $f$ and $\Delta_{c} f$ share $\{a,-a\} C M$, then $\Delta_{c} f(z)=f(z)$ for all $z \in \mathbb{C}$.

Liu also proposed the question as follows [2]: Let $a$ and $b$ be two small functions of $f$ with period $c$. When a transcendental entire function $f$ of finite order and its difference operator $\Delta_{c} f$ share the set $\{a, b\} \mathrm{CM}$, what can we say about the relationship between $f$ and $\Delta_{f} f$ ?

In 2012, Li [1] considered the problem and proved the following.

Theorem B Suppose that $a, b$ are two distinct entire functions, and $f$ is a nonconstant entire function with $\rho(f) \neq 1$ and $\lambda(f)<\rho(f)<\infty$ such that $\rho(a)<\rho(f)$ and $\rho(b)<\rho(f)$. If $f$ and $\Delta_{c} f$ share $\{a, b\} C M$, then $f(z)=\Delta_{c} f(z)$ for all $z \in \mathbb{C}$.

Here, the order $\rho(f)$ is defined by

$$
\rho(f)=\limsup _{r \rightarrow \infty} \frac{\log T(r, f)}{\log r},
$$

and the exponent of convergence of zeros $\lambda(f)$ is defined by

$$
\lambda(f)=\limsup _{r \rightarrow \infty} \frac{\log N\left(r, \frac{1}{f}\right)}{\log r} .
$$

In Theorem B, the condition $\rho(f) \neq 1$ seems not natural. So one may ask whether it can be removed or not. In this paper, we consider the question and show that the theorem still holds without the condition $\rho(f) \neq 1$. More precisely, we give the specific form of $f$ in a simple way.

Main theorem Suppose that $a, b$ are two distinct entire functions, and $f$ is a nonconstant entire function of finite order with $\lambda(f)<\rho(f)<\infty$ such that $\rho(a)<\rho(f)$ and $\rho(b)<\rho(f)$. Iff and $\Delta_{c} f$ share $\{a, b\} C M$, then $f(z)=A e^{\mu z}$, where $A, \mu$ are two nonzero constants satisfying $e^{\mu c}=2$. Furthermore, $f(z)=\Delta_{c} f(z)$.

Using the almost same method, we generalize the above result from entire functions to meromorphic functions after the above theorem. Due to the use of almost the same method, we omit the detailed proof.

Corollary Suppose that $a, b$ are two distinct entire functions, and $f$ is a nonconstant $f$ nite order meromorphic function which has finite poles and with $\lambda(f)<\rho(f)<\infty$ such that $\rho(a)<\rho(f)$ and $\rho(b)<\rho(f)$. Iff and $\Delta_{c} f$ share $\{a, b\} C M$, then $f(z)=A e^{\mu z}$, where $A, \mu$ are two nonzero constants satisfying $e^{\mu c}=2$. Furthermore, $f(z)=\Delta_{c} f(z)$.

A similar result can be found in [11]. Before we proceed, we suppose that the reader is familiar with Nevanlinna theory, for example, the first and second main theorems, and the 
common notations such as $T(r, f), m(r, f)$ and $N(r, f) . S(r, f)$ denotes any quantity which satisfies $S(r, f)=o(T(r, f))$ as $r \rightarrow \infty$, except possibly on a set of finite logarithmic measure; see e.g. [12-14].

\section{Proof of Main theorem}

We will prove our theorem in this section. Before we turn to its proof, we first give the following results, where the first one is Corollary 2.6 of Chiang and Feng in [10], and the second one is Lemma 3.3 of Bergweiler and Langley in [15].

Lemma 2.1 Letf be a meromorphic function of finite order and let $\omega_{1}, \omega_{2}$ be two arbitrary complex numbers such that $\omega_{1} \neq \omega_{2}$. Assume that $\sigma$ is the order off, then for each $\epsilon>0$, we have

$$
m\left(r, \frac{f\left(z+\omega_{1}\right)}{f\left(z+\omega_{2}\right)}\right)=O\left(r^{\sigma-1+\epsilon}\right) .
$$

Lemma 2.2 Let $g$ be a function transcendental and meromorphic in the plane of order less than 1 . Set $h>0$. Then there exists an $\epsilon$-set $E$ such that

$$
\frac{g(z+\omega)}{g(z)} \rightarrow 1, \quad \text { when } z \rightarrow \infty \text { in } \mathbb{C} \backslash E,
$$

uniformly in $\omega$ for $|\omega| \leq h$.

Proof of main theorem Note that $f$ and $\Delta_{c} f$ share $\{a, b\}$ CM. So we can set

$$
\frac{\left(\Delta_{c} f-a\right)\left(\Delta_{c} f-b\right)}{(f-a)(f-b)}=e^{Q}
$$

in which $Q$ is an entire function. Furthermore, it follows from (2.1) and $\max \{\rho(a), \rho(b)\}<$ $\rho(f)<\infty$ that $Q$ is a polynomial.

By the Hadamard factorization theorem, we suppose that $f(z)=h(z) e^{P(z)}$, where $h(\not \equiv 0)$ is an entire function and $P$ is a polynomial satisfying

$$
\lambda(f)=\rho(h)<\rho(f)=\operatorname{deg}(P) .
$$

Then

$$
\Delta_{c} f=f(z+c)-f(z)=\left(h(z+c) e^{P(z+c)-P(z)}-h(z)\right) e^{P(z)} .
$$

We substitute the forms of $f$ and $\Delta_{c} f$ into (2.1) to find

$$
\begin{aligned}
& {\left[\left(h(z+c) e^{P(z+c)-P(z)}-h(z)\right) e^{P(z)}-a(z)\right]\left[\left(h(z+c) e^{P(z+c)-P(z)}-h(z)\right) e^{P(z)}-b(z)\right]} \\
& \quad=\left(h(z) e^{P(z)}-a(z)\right)\left(h(z) e^{P(z)}-b(z)\right) e^{Q(z)} .
\end{aligned}
$$

Set $w_{1}=\left(h(z+c) e^{P(z+c)-P(z)}-h(z)\right)$. Suppose that $w_{1} \equiv 0$. Then,

$$
h(z+c) e^{P(z+c)}=h(z) e^{P(z)},
$$


which means $\Delta_{f} f \equiv 0$. It contradicts with Eq. (2.1). Thus, we suppose that $w_{1} \not \equiv 0$ below. Obviously, $w_{1}$ is a small function of $e^{P}$. Rewrite (2.2) as

$$
e^{Q}=\frac{w_{1}^{2}\left[e^{P}-\frac{a}{w_{1}}\right]\left[e^{P}-\frac{b}{w_{1}}\right]}{h^{2}\left[e^{P}-\frac{a}{h}\right]\left[e^{P}-\frac{b}{h}\right]} .
$$

Note that $a \not \equiv b$. Without loss of generality, we suppose that $a \not \equiv 0$. Assume that $z_{0}$ is a zero of $e^{P}-\frac{a}{h}$, but not a zero of $w_{1}$. It follows from (2.3) and the assumption about sharing that $z_{0}$ is a zero of $e^{P}-\frac{a}{w_{1}}$ or $e^{P}-\frac{b}{w_{1}}$. We denote by $N_{1}\left(r, e^{P}\right)$ the reduced counting function of those common zeros of $e^{P}-\frac{a}{h}$ and $e^{P(z)}-\frac{a}{w_{1}}$. Similarly, we denote by $N_{2}\left(r, e^{P}\right)$ the reduced counting function of those common zeros of $e^{P}-\frac{a}{h}$ and $e^{P}-\frac{b}{w_{1}}$. Note that $h$ is a small function respect to $e^{P}$; applying the second fundamental theorem to $e^{P}$ and the first fundamental theorem to $e^{P}-\frac{a}{h}$ yields

$$
T\left(r, e^{P}\right)=\bar{N}\left(r, \frac{1}{e^{P(z)}-\frac{a}{h}}\right)+S\left(r, e^{P}\right)=N_{1}\left(r, e^{P}\right)+N_{2}\left(r, e^{P}\right)+S\left(r, e^{P}\right),
$$

which implies that either $N_{1}\left(r, e^{P}\right) \neq S\left(r, e^{P}\right)$ or $N_{2}\left(r, e^{P}\right) \neq S\left(r, e^{P}\right)$. We consider the following two cases.

Case 1. $N_{1}\left(r, e^{P}\right) \neq S\left(r, e^{P}\right)$.

Let $a_{0}$ be a common zero of $e^{P}-\frac{a}{h}$ and $e^{P}-\frac{a}{w_{1}}$. Then it is clear that $a_{0}$ is a zero of $\frac{a}{h}-\frac{a}{w_{1}}$. If $\frac{a}{h}-\frac{a}{w_{1}} \not \equiv 0$, then

$$
S\left(r, e^{P}\right) \neq N_{1}\left(r, e^{P}\right) \leq N\left(r, \frac{1}{\frac{a}{h}-\frac{a}{w_{1}}}\right) \leq T\left(r, \frac{a}{h}-\frac{a}{w_{1}}\right)=S\left(r, e^{P}\right),
$$

a contradiction. Thus

$$
h \equiv w_{1} .
$$

It leads to

$$
2 \frac{h(z)}{h(z+c)}=e^{P(z+c)-P(z)} .
$$

Then, by Lemma 2.1, one has, for any $\epsilon>0$,

$$
m\left(r, e^{P(z+c)-P(z)}\right)=m\left(r, \frac{h(z)}{h(z+c)}\right)+O(1)=O\left(r^{\rho(h)-1+\epsilon}\right)+O(1) .
$$

On the other hand, $m\left(r, e^{P(z+c)-P(z)}\right)=[A+o(1)] r^{\rho(f)-1}$, where $A$ is a fixed positive constant.

If $\rho(f)>1$, by $\rho(f)>\rho(h)$ and the above estimates of $m\left(r, e^{P(z+c)-P(z)}\right)$, we can easily get a contradiction. So, $\rho(f) \leq 1$, which means that $e^{P(z+c)-P(z)}$ is a nonzero constant, say $c_{0}$. Then (2.6) reduces to

$$
2 \frac{h(z)}{h(z+c)}=c_{0}
$$


Note that $1 \geq \rho(f)>\rho(h)$. Then by Lemma 2.2, we know that there exists an $\epsilon$-set $E$, as $z \notin E$ and $|z| \rightarrow \infty$ such that

$$
\frac{h(z+c)}{h(z)} \rightarrow 1
$$

So, $c_{0}=2$ and $h(z)=h(z+c)$, which means that $h$ is a periodic function. If $h$ is a nonconstant function, then $\rho(h) \geq 1$, a contradiction. Therefore, $h$ is a constant. Note that $\operatorname{deg}(P)=$ $\rho(f) \leq 1$ and $f$ is a nonconstant entire function. Then $\operatorname{deg}(P)=1$. Thus, we can $\operatorname{set} f=A e^{\mu z}$, where $A, \mu$ are two nonzero constants.

By the assumption of Case 1 , one sees that $f-a$ and $\Delta_{c} f-a$ have common zeros, which are not zeros of $a$. Assume that $\alpha_{0}$ is a common zero of $f-a$ and $\Delta_{c} f-a$, and not a zero of $a$. Then $z_{0}$ is a zero of $f(z+c)-2 a(z)$. Furthermore,

$$
A e^{\mu \alpha_{0}}-a\left(\alpha_{0}\right)=0, \quad A e^{\mu c} e^{\mu \alpha_{0}}-2 a\left(\alpha_{0}\right)=0,
$$

which implies that $e^{\mu c}=2$. Thus, we get $\Delta_{c} f=f$, which is the desired result.

Case 2. $N_{2}\left(r, e^{P}\right) \neq S\left(r, e^{P}\right)$.

Let $b_{0}$ be a common zero of $e^{P}-\frac{a}{h}$ and $e^{P}-\frac{b}{w_{1}}$. Then it is clear that $b_{0}$ is a zero of $\frac{a}{h}-\frac{b}{w_{1}}$. If $\frac{a}{h}-\frac{b}{w_{1}} \not \equiv 0$, then

$$
S\left(r, e^{P}\right) \neq N_{2}\left(r, e^{P}\right) \leq N\left(r, \frac{1}{\frac{a}{h}-\frac{b}{w_{1}}}\right) \leq T\left(r, \frac{a}{h}-\frac{b}{w_{1}}\right)=S\left(r, e^{P}\right),
$$

a contradiction. Thus

$$
\frac{a}{h}-\frac{b}{w_{1}} \equiv 0
$$

If $b \equiv 0$, then $\frac{a}{h} \equiv 0$, a contradiction. Thus, $b \neq 0$.

We assume that $c_{0}$ is a zero of $e^{P}-\frac{b}{h}$, but not a zero of $w_{1}$. It follows from (2.3) that $c_{0}$ is a zero of $e^{P}-\frac{a}{w_{1}}$ or $e^{P}-\frac{b}{w_{1}}$. We denote by $N_{3}\left(r, e^{P}\right)$ the reduced counting function of those common zeros of $e^{P}-\frac{b}{h}$ and $e^{P}-\frac{a}{w_{1}}$. Similarly, we denote by $N_{4}\left(r, e^{P}\right)$ the reduced counting function of those common zeros of $e^{P}-\frac{b}{h}$ and $e^{P}-\frac{b}{w_{1}}$. Then

$$
T\left(r, e^{P}\right)=\bar{N}\left(r, \frac{1}{e^{P}-\frac{b}{h}}\right)+S\left(r, e^{P}\right)=N_{3}\left(r, e^{P}\right)+N_{4}\left(r, e^{P}\right)+S\left(r, e^{P}\right)
$$

which implies that either $N_{3}\left(r, e^{P}\right) \neq S\left(r, e^{P}\right)$ or $N_{4}\left(r, e^{P}\right) \neq S\left(r, e^{P}\right)$. If $N_{4}\left(r, e^{P}\right) \neq S\left(r, e^{P}\right)$, similar to Case 1 , we get the desired result. So, we assume that $N_{3}\left(r, e^{P}\right) \neq S\left(r, e^{P}\right)$ below. Similar to Case 2, we can deduce that

$$
\frac{b}{h}-\frac{a}{w_{1}} \equiv 0
$$

It follows from (2.7) and (2.9) that

$$
a^{2}=b^{2} \text {. }
$$


Note that $a \neq \equiv b$. Thus, $a \equiv-b$. Again by (2.9), one has $w_{1}=-h$. We can rewrite it as

$$
h(z+c) e^{P(z+c)-P(z)} \equiv 0
$$

\section{a contradiction.}

Therefore, the proof of the main theorem is finished.

\section{Acknowledgements}

The authors wish to thank the editors and referees for their great useful suggestions and helpful comments.

\section{Funding}

This work was supported by Applied Mathematical Academic Discipline Project of Shanghai Dianji University (16JCXK02), and Humanity and Social Science Youth foundation of Ministry of Education(18YJC630120) and NNSF of China Project (11601521), and the Fundamental Research Fund for Central Universities in China Project (15CX05061A, 15CX05063A 15CX08011A).

\section{Competing interests}

The authors declare that they have no competing interests.

\section{Authors' contributions}

All authors typed, read and approved the final manuscript.

\section{Author details}

${ }^{1}$ Business College, Shanghai Dianji University, Shanghai, P.R. China. ${ }^{2}$ Department of Physics and Mathematics, University of Eastern Finland, Joensuu, Finland. ${ }^{3}$ College of Science, China University of Petroleum, Qingdao, P.R. China. ${ }^{4}$ School of Mathematics and Information Science, Guangzhou University, Guangzhou, P.R. China.

\section{Publisher's Note}

Springer Nature remains neutral with regard to jurisdictional claims in published maps and institutional affiliations.

Received: 7 April 2018 Accepted: 27 January 2019 Published online: 19 March 2019

\section{References}

1. Li, X.M.: Entire functions sharing a finite set with their difference operators. Comput. Methods Funct. Theory 12 $307-328(2012)$

2. Liu, K.: Meromorphic functions sharing a set with applications to difference equation. J. Math. Anal. Appl. 359 384-393 (2009)

3. Gross, F., Yang, C.C.: On preimage range sets of meromorphic functions. Proc. Jpn. Acad., Ser. A 58, 17-20 (1982)

4. Gross, F.: Factorization of meromorphic functions and some open problems. In: Complex Analysis (Proc. Conf., Univ. Kentucky, Lexington, Ky., 1976). Lecture Notes in Math., vol. 599, pp. 51-69. Springer, Berlin (1977)

5. Yi, H.X.: A question of Gross and the uniqueness of entire function. Nagoya Math. J. 138, 169-177 (1995)

6. Frank, G., Reinders, M.: A unique range set for meromorphic functions with 11 elements. Complex Var. Theory Appl. 37, 185-193 (1998)

7. Li, P., Yang, C.C.: Some further results on the unique range sets of meromorphic functions. Kodai Math. J. 18, 437-450 (1995)

8. Li, P., Yang, C.C.: Value sharing of an entire function and its derivatives. J. Math. Soc. Jpn. 51, 781-799 (1999)

9. Fang, M.L., Zalcman, L.: Normal families and uniqueness theorems for entire functions. J. Math. Anal. Appl. 280, 273-283 (2003)

10. Chiang, Y.M., Feng, S.J.: On the Nevanlinna characteristic of $f(z+\eta)$ and difference equations in the complex plane. Ramanujan J. 16, 105-129 (2008)

11. Chen, B.Q., Chen, Z.X.: Entire functions sharing sets of small functions with their difference operators or shifts. Math. Slovaca 63, 1233-1246 (2013)

12. Hayman, W.K.: Meromorphic Functions. Clarendon, Oxford (1964)

13. Laine, I: Nevanlinna Theory and Complex Differential Equations, de Gruyter, Berlin (1993)

14. Yang, C.C., Yi, H.X.: Uniqueness Theory of Meromorphic Functions. Science Press, Beijing (2003)

15. Bergweiler, W., Langley, J.K.: Zeros of differences of meromorphic functions. Math. Proc. Camb. Philos. Soc. 142 133-147 (2007)

16. Chiang, Y.M., Feng, S.J.: Nevanlinna theory of the Askey-Wilson divided difference operator. Adv. Math. 329, 217-272 (2018)

17. Halburd, R.G., Korhonen, R.J.: Nevanlinna theory for the difference operators. Ann. Acad. Sci. Fenn., Math. 31(2), 463-478 (2006)

18. Halburd, R.G., Korhonen, R.J.: Difference analogue of the lemma on the logarithmic derivative with applications to difference equations. J. Math. Anal. Appl. 314(2), 477-487 (2006)

19. Laine, I., Yang, C.C.: Clunie theorems for difference and q-difference polynomials. J. Lond. Math. Soc. 76, 556-566 (2007)

20. Li, S., Chen, B.Q.: Results on meromorphic solutions of linear difference equations. Adv. Differ. Equ. 2012, 203 (2012) 
21. Li, S., Mei, D., Chen, B.Q.: Uniqueness of entire functions sharing two values with their difference operators. Adv. Differ. Equ. 2017, 390 (2017)

22. Li, S., Mei, D., Chen, B.Q.: Meromorphic functions sharing small functions with their linear difference polynomials. Adv. Differ. Equ. 2013, 58 (2013)

23. Chen, B.Q., Li, S.: Uniqueness problems on entire functions that share a small function with their difference operators. Adv. Differ. Equ. 2014, 311 (2014)

24. Fujimoto, H.: On uniqueness polynomials for meromorphic functions. Nagoya Math. J. 170, 33-46 (2003)

Submit your manuscript to a SpringerOpen ${ }^{\odot}$ journal and benefit from:

- Convenient online submission

- Rigorous peer review

- Open access: articles freely available online

- High visibility within the field

- Retaining the copyright to your article

Submit your next manuscript at $\gg$ springeropen.com 